\title{
Dissolution Fit Factors as Response Variables in Statistically Designed Experiments
}

\author{
Henry H. Flanner, ${ }^{* 1}$ Charles F. Vesey, ${ }^{2}$ and Joseph R. Loehe, ${ }^{3}$ \\ 1 Drug Delivery Sciences, Shire Laboratories, Inc., Rockville, MD \\ 2 New Product Development, Colorcon, West Point, PA \\ ${ }^{3}$ Consulting Chemical Engineer, Ellicott City, MD
}

email:hflanner@shirelabs.com

*to whom correspondence should be addressed

\section{Introduction}

ince the introduction of the $f_{2}$ metric in 1994 and its subsequent inclusion in various guidances, the use of the $\mathrm{f}_{2}$ metric for dissolution profile comparison has become commonplace in the industry. Other metrics have been considered and compared with $f_{2}(1)$.The $f_{2}$ metric has been studied extensively for its statistical attributes (2), and the shortcomings of this metric have been pointed out (3). However, most of the literature regarding dissolution profile comparison has focused on the regulatory applications of these metrics. For the pharmaceutical formulator, the benefit of dissolution profile comparison lies in the ability to accurately distinguish between formulations. During development, the formulator has to evaluate the effect of various formulation and process variables on the dissolution profile.The effect of these variables on dissolution is difficult to determine by inspection, so the dissolution metric potentially could become a valuable tool for this purpose. However, the suitability of $\mathrm{f}_{2}$ and other metrics as response variables in statistically designed experiments has not been considered thoroughly.

An ideal response variable should provide a reproducible and objective means of assessing the effect of changing the controlled variable levels. In addition, the response variable should have statistical properties that permit it to be modeled appropriately by the empirical functions normally associated with statistically designed experiments. If the metrics have appropriate statistical properties, it will be possible to estimate the model coefficients without bias. If they do not, some of the model terms will not reflect the cause and effect relationships between the controlled variables and reponses essential to efficient product development.Indeed, research conducted at Shire Laboratories, Inc. (SLI) using small factorial designs (4-8 design points) has shown that $\mathrm{f}_{1}$ and $\mathrm{f}_{2}$ behave differently when used as response variables. Often, significant factors and interactions for the best-fit mathematical models of the two metrics in the same experiment are quite different, and $R^{2}$ values and lack-of-fit statistics vary widely between them. Due to the small size of the experiments run at SLI the importance of these observed differences cannot be studied thoroughly.

Therefore, it is the object of this paper to investigate the use of $f_{1}$ and $f_{2}$ as response variables in a larger statistically designed experiment.The significant factors and interactions as well as the mathematical models developed from the design will be compared. Mathematical models derived for both metrics will be compared and analyzed for robustness and precision by examining $\mathrm{f}_{2}$, lack of fit, prediction accuracy, and residuals.

\section{Experimental}

A 30 run D-optimal experimental design was conducted at Colorcon,Inc. to evaluate the effect of various factors on the dissolution profile of Surelease ${ }^{\circledR}$ coated pellets (4).The experimental design considered the effects of Inlet Air Temperature, Atomizing Air Pressure, Spray Rate, and Coating Dispersion Solids Content. In order to compare the $f_{1}$ and $\mathrm{f}_{2}$ metrics as response variables, the original $\mathrm{D}$ optimal design was broken into a nearly complete 4 factor 2 level full factorial design with two center points, see Table 1 (page 14) for the factorial experimental design. The remaining 12 experiments from the D-optimal design were used to check the accuracy of the model developed from the full factorial design.

The experiment was designed and conducted by Colorcon personnel.The original purpose was to study the effect of the variables tested on the curing properties of Surelease ${ }^{\circledR}$. This work was presented in a poster at the AAPS Annual meeting in 1999.In order to generate $f_{1}$ and $f_{2}$ data for this article a "target" profile was generated to serve as the reference profile.

In formulation development of new controlled release dosage forms, an imaginary"target" profile is frequently utilized to help direct the formulation effort.This target profile often shifts as the project progresses and more is learned about the absorption of the compound from in vivo studies in animals or humans. In development of generic controlled release (or immediate release) products the innovator product serves as the reference profile. In this experiment the reference profile remained constant. Dissolution profiles from each experimental run served as the test profile in the $f_{1}$ and $f_{2}$ calculations. 


\section{Dissolution Fit Factors ... continued}

The experimental design was analyzed using Statgraphics software. Significant factors and interactions were determined by analysis of variance (ANOVA). Mathematical models were derived from the significant factors and interactions and tested for lack of fit, and $r^{2}$.To avoid overfitting the data, the models were kept to as few terms as possible while keeping an acceptable goodness of fit. The residuals were examined for any abnormalities in the model predictions and fit.

\section{Results and Discussion}

The maximum order effect considered in analyzing the design and developing the model was $2^{\text {nd }}$ order. The higher order effects were confounded because of the missing data from run 24. Using the higher order effects to estimate the error, an analysis of variance was conducted to determine which main effects and 2 factor interactions were significant. Results from the ANOVA for both $f_{1}$ and $f_{2}$ are presented in Table 2 (on page 16).

The ANOVA indicated that both $f_{1}$ and $f_{2}$ predict the same significant main effect, Spray Rate, and interaction,Spray Rate with \% Solids. Therefore the model for both response variables consists of three terms:

- Spray Rate,

- \% Solids and the

- interaction Spray Rate*\% Solids.
For $\mathrm{f}_{1}$ the equation is: $f_{1}=46.9-1.4^{*}$ SprayRate $-2.5^{*} \%$ Solids + $0.1 *$ SprayRate $\%$ Solids

For $\mathrm{f} 2$ the equation is:

$f_{2}=22.9+1.3^{*}$ SprayRate $+2.6 * \%$ Solids -

$0.1 *$ SprayRate*\%Solids.

The lack-of-fit for both response variables was not significant. The $r^{2}$ of the $f_{1}$ model was 0.56 , while the $r^{2}$ of the $f_{2}$ model was 0.63 . These $r^{2}$ values are low because there are only 3 significant terms in the model to describe 17 data points.

Inspection of Table 2 reveals that both response variables rank ordered the main effects similarly; however, the rank order correlation was lost when considering interactions. Interaction $A B$ is borderline significant in the analysis with $\mathrm{f}_{1}$, and may need to be considered in the model, but $A B$ is not significant when $\mathrm{f}_{2}$ is the response variable. The difference in prediction of significant interactions by the two response variables is illustrated in Figures 1 and 2 .

Close examination of the interaction plots indicates that the two response variables are affected by interactions differently. Particularly interesting interactions are $B C$ and $C D$. In Figure $1 B C$ and $C D$ are parallel,indicating virtually no interaction between the factors, but in Figure 2 it is clear there is a slight interaction. This is also reflected in the probabilities from the ANOVA in Table 2. While there was no

\section{Table 1. Experimental Design}

\begin{tabular}{|ccccccc|}
\hline Test Run \# & Inlet $\left.\mathbf{~}^{\circ} \mathbf{C}\right)$ & $\begin{array}{c}\text { Spray Rate } \\
(\mathbf{g} / \mathbf{m i n})\end{array}$ & $\begin{array}{c}\text { Atomization } \\
\text { Air (Bar) }\end{array}$ & $\begin{array}{c}\text { Solids } \\
\text { Content (\%) }\end{array}$ & $\begin{array}{c}\text { Response } \\
\text { (f1) }\end{array}$ & $\begin{array}{c}\text { Response } \\
\text { (f2) }\end{array}$ \\
\hline 1 & 50 & 15 & 3 & 10 & 24 & 47 \\
2 & 50 & 15 & 1 & 10 & 14 & 60 \\
4 & 70 & 45 & 1 & 25 & 42 & 38 \\
5 & 70 & 45 & 1 & 10 & 13 & 61 \\
6 & 50 & 45 & 1 & 25 & 56 & 32 \\
10 & 70 & 15 & 1 & 10 & 16 & 55 \\
11 & 50 & 15 & 3 & 25 & 12 & 62 \\
13 & 60 & 30 & 2 & 17.5 & 8 & 72 \\
14 & 70 & 45 & 3 & 25 & 21 & 52 \\
15 & 70 & 15 & 3 & 10 & 26 & 47 \\
16 & 70 & 15 & 3 & 25 & 10 & 64 \\
17 & 70 & 15 & 1 & 25 & 3 & 88 \\
18 & 50 & 45 & 3 & 25 & 99 & 20 \\
22 & 60 & 30 & 2 & 17.5 & 13 & 61 \\
24 & 50 & 45 & 3 & 10 & $*$ & $*$ \\
25 & 70 & 45 & 3 & 10 & 9 & 69 \\
28 & 50 & 15 & 1 & 25 & 10 & 65 \\
29 & 50 & 45 & 1 & 10 & 21 & 52 \\
•indicates test conditions inadequate to produce product. & & & & \\
\hline
\end{tabular}


Table 2. ANOVA Probabilities for $\mathrm{f} 1$ and $\mathrm{f} 2$

\begin{tabular}{|lcc|}
\hline Source & Probability,f1 & Probability, f2 \\
\hline A:Inlet & 0.11 & 0.31 \\
B:Spray Rate & 0.02 & 0.049 \\
C:Atm. Air & 0.21 & 0.37 \\
D:Solids & 0.40 & 0.79 \\
AB & 0.053 & 0.16 \\
AC & 0.13 & 0.35 \\
AD & 0.69 & 0.90 \\
BC & 0.96 & 0.20 \\
BD & 0.02 & 0.009 \\
CD & 0.98 & 0.65 \\
\hline
\end{tabular}

difference in the significant factors and interactions in this example, it is clear that had the standard error been smaller, from tighter data or replication of the design, the two response variables, $\mathrm{f}_{1}$ and $\mathrm{f}_{2}$, would have predicted different significant interactions even though they are both simple measures of the distance between two curves.

An important clue to the underlying differences between the models for the response variables is revealed by residuals analysis. The residuals (observed minus predicted values) were graphed versus various parameters such as time sequence, individual factors, and the predicted value to assist in visualization of trends that indicate a potential problem with the model. Of particular interest is the residuals versus predicted response graph for both response variables, shown in Figures 3 and 4 (page 17).

In Figure 3 the funnel shape of the residuals indicates that the prediction error is not constant, i.e.the error increases with increasing values of $\mathrm{f1}$. This feature combined with a slight curvilinear trend in the residuals of Figure 3 suggests a transformation of the response variable is in order. The residual plot of Figure 4 shows the residuals for the $\mathrm{f}_{2}$ model equation are randomly distributed, as they should be.

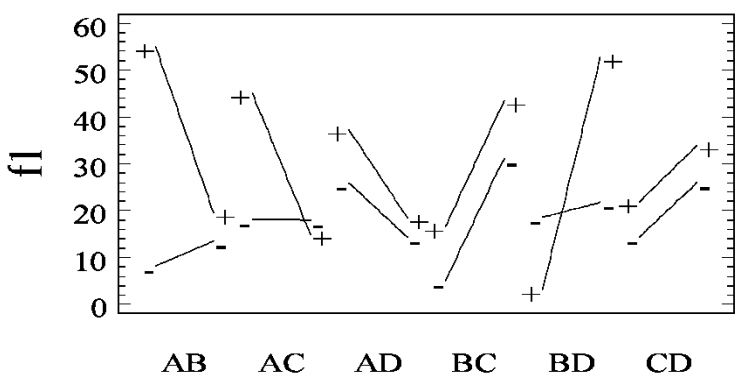

Figure 1: Interaction Plot for f1
Table 3. ANOVA Probabilities for $\log f_{1}$

\begin{tabular}{|lc|}
\hline Source & Probability, log $\mathbf{f 1}$ \\
\hline A:Inlet & 0.33 \\
B:Spray Rate & 0.053 \\
C:Atm. Air & 0.42 \\
D:Solids & 0.82 \\
AB & 0.23 \\
AC & 0.43 \\
AD & 0.84 \\
BC & 0.22 \\
BD & 0.01 \\
CD & 0.69 \\
\hline
\end{tabular}

For this experimental design, $\mathrm{f}_{2}$ fits the experimental data better than $f_{1}$. While there can be no certainty that this will always be the case, there are statistical reasons to believe that $f_{2}$ may turn out to be a more useful response variable for other test plans as well. We intend to investigate this issue in future work.

A log transformation of $\mathrm{f} 1$ was made and evaluated as a response variable in this experimental design. Results from the logf1 ANOVA are given in Table 3.The only significant factor detected was the $\mathrm{BD}$ interaction.Therefore, the model for logf1 contains the same three terms as the other models.

The equation is:

logf $1=1.9-0.03 *$ SprayRate $-0.06 * \%$ Solids $+0.002 *$ SprayRate*\%Solids.

The $r^{2}$ was 0.63 and the lack-of-fit was insignificant. Comparison of Table 3 with Table 2 indicates much closer agreement of $\log f_{1}$ with $f_{2}$ in prediction of significant interactions. As a whole, the relative magnitude of the probabilities of logf 1 are in close agreement with those of $f_{2}$. The $\log f_{1} r^{2}$ value of 0.63 is identical to that of $f_{2}$ and improved over the untransformed $f_{1}$. Examination of the interac-

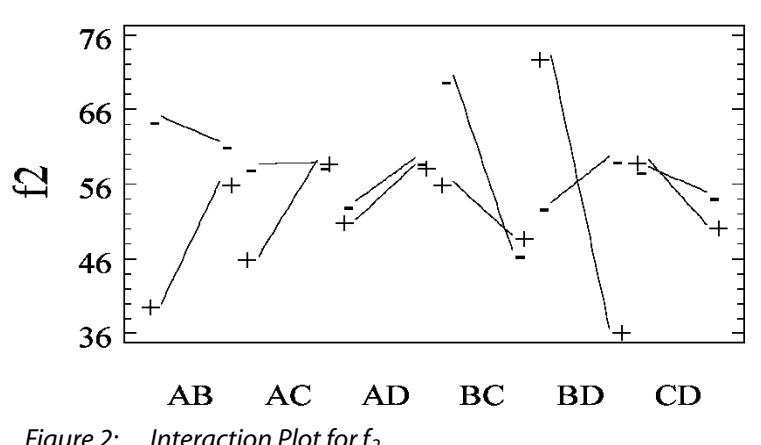

Figure 2: Interaction Plot for $\mathrm{f}_{2}$ 


\section{Dissolution Fit Factors ... continued}

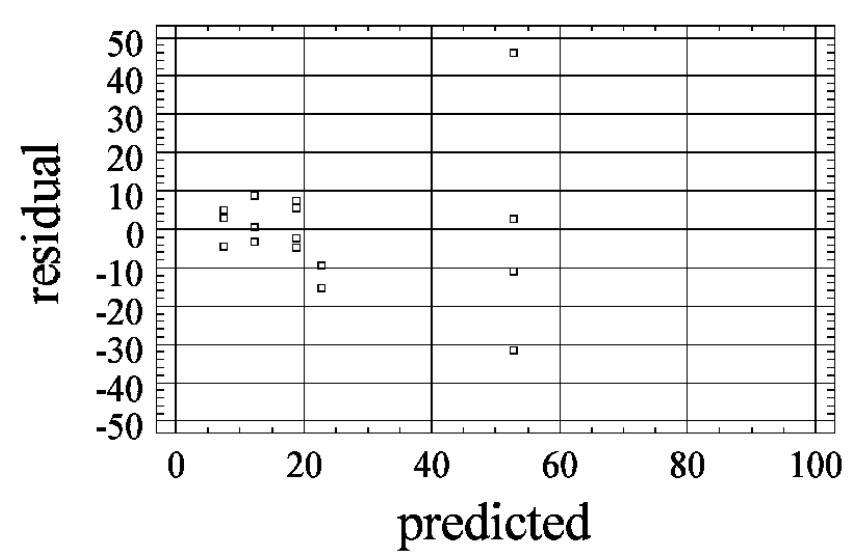

Figure 3: Residuals vs. Predicted $f_{1}$

tion plot in Figure 5 also indicates close agreement with the $\mathrm{f}_{2}$ interaction plot of Figure 2. Most notably, interactions $B C$ and $C D$ are no longer parallel after the log transformation of $f_{1}$.

The residual plot of $\log \left(f_{1}\right)$ also shows none of the curvilinear or funnel shape evident in Figure 3. A normal probability plot of the residuals confirms the observation, that the residuals for $\log \left(f_{1}\right)$ are normally distributed (Figure 6 ). The log-transformed $f_{1}$ appears to fit the observations from the experimental design much better than $\mathrm{f}_{1}$ in this case.

The improved fit of $\log \left(f_{1}\right)$ can be explained by the mathematical relationship between $\mathrm{f}_{1}$ and $\mathrm{f}_{2}$. Both are measures of distance between two curves, and both utilize the common term, $R_{t}-T_{t}$, to represent the distance. In fact, the two dissolution comparison metrics, $\mathrm{f}_{1}$ and $\mathrm{f}_{2}$, can be expressed in terms of each other. In this experiment $\log \left(f_{1}\right)$ can be adequately described in terms of $f_{2}$ by the simple non-linear model $\log \left(f_{1}\right)=5.677-0.051 * f_{2}$. The relationship is depicted graphically in Figure 7 (page 18). The $\mathrm{r}^{2}$ for this equation was 0.995 .

The implication here is that the choice of dissolution fit factor in the modeling of complex systems must not be taken lightly.The model developed must be thoroughly

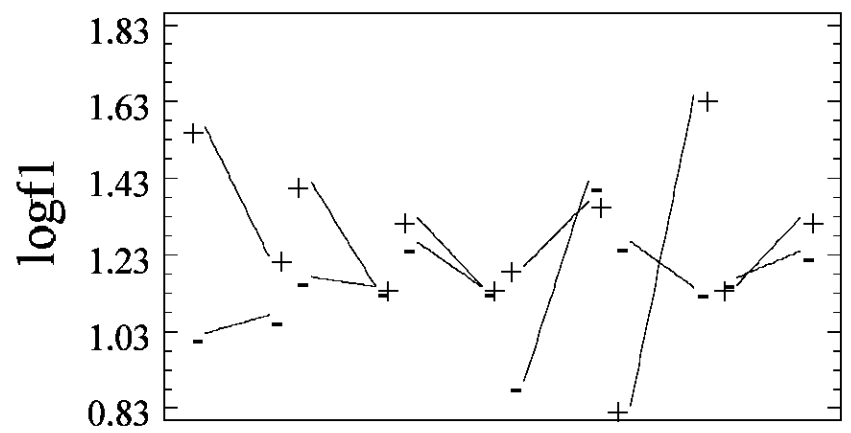

$$
\mathrm{AB} \quad \mathrm{AC} \quad \mathrm{AD} \quad \mathrm{BC} \quad \mathrm{BD} \quad \mathrm{CD}
$$

Figure 5: Interaction Plot of $\log (f 1)$

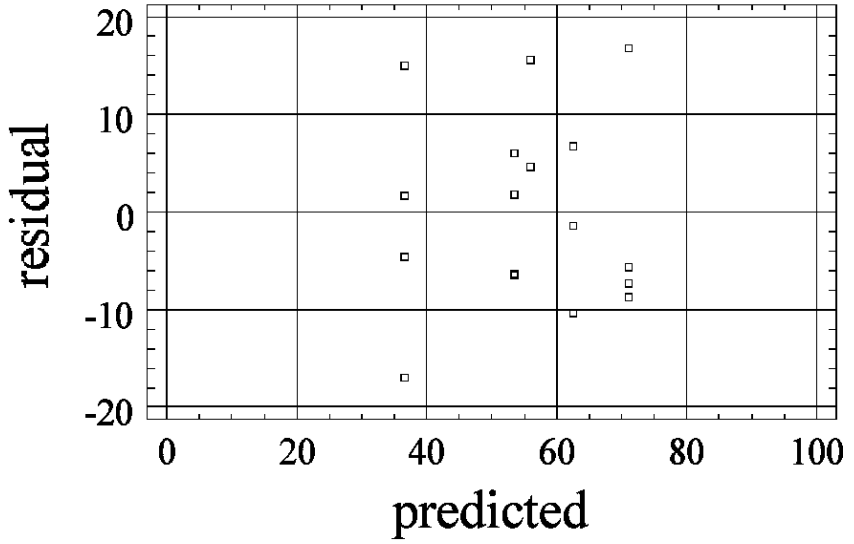

Figure 4: Residuals vs. Predicted $f_{2}$

tested to insure the predictions are accurate. For the development scientist conducting early formulation and process studies, the impact of following the wrong path can lead to project delays, wasted resources, a poorly or incorrectly optimized formula and general confusion over the data. For the production engineer conducting evolutionary operations (EVOP) on the plant floor to make slight improvements to product yield or performance, the impact of developing and following a poor model can be wasted resources, out of specification product, recalls and confusion over the process possibly resulting in a SUPAC submission error.

\section{Conclusions}

In the analysis of this experimental design, it was clearly shown that the fit factors, $f_{1}$ and $f_{2}$, do not function equally as response variables even though both fit factors provide simple measures of the relative distance between two profiles. The use of these fit factors as response variables in statistically designed experiments should be considered carefully and any models developed must be tested fully. Failure to fully test models developed with these fit factors

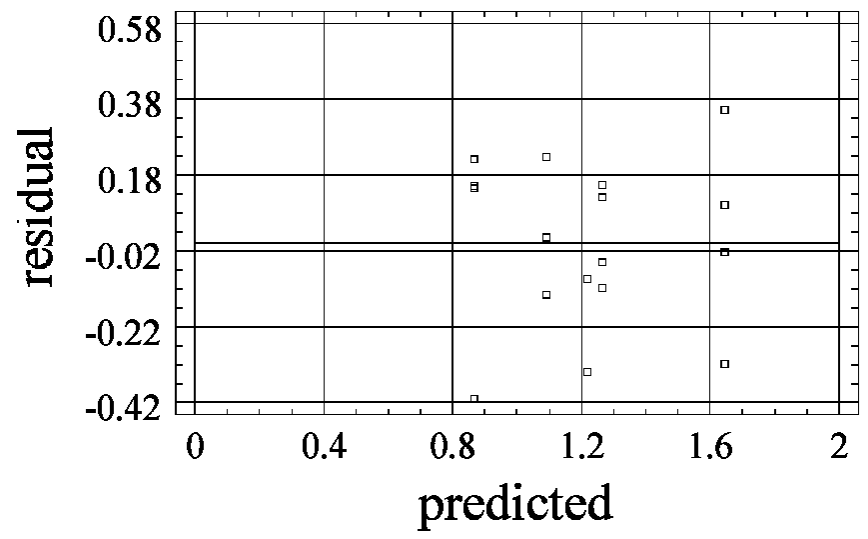

Figure 6: Residuals vs. Predicted, $\log \left(f_{1)}\right.$ 


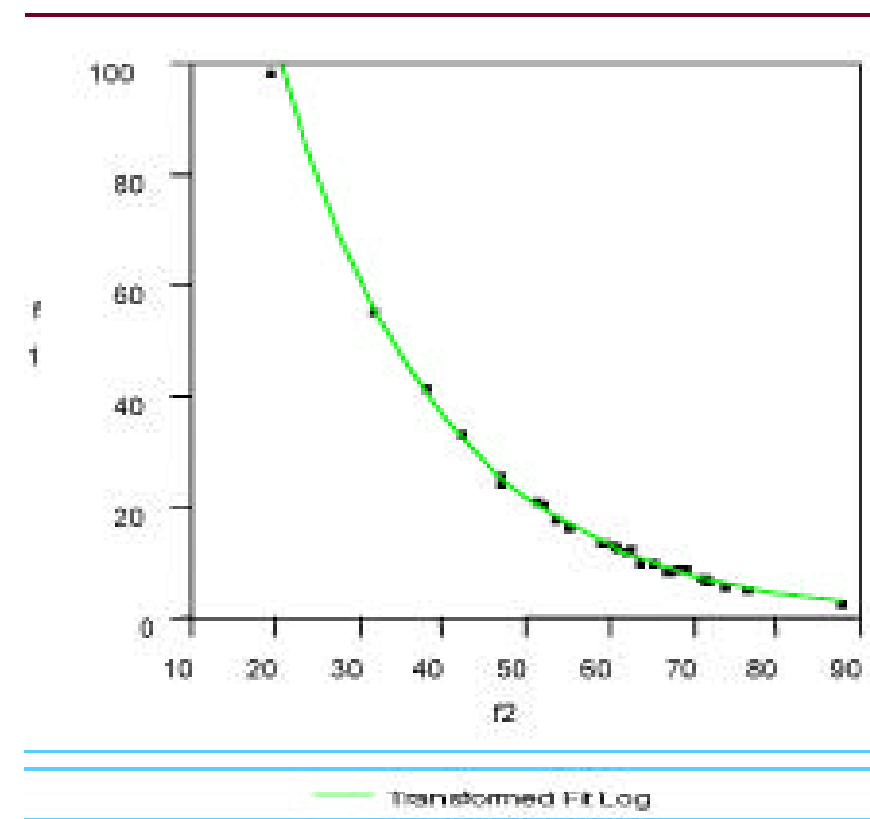

Figure 7: Relationship Between $f_{1}$ and $f_{2}$

could lead to drastic consequences for the development scientist or production engineer. Due to the relationship between $\mathrm{f} 1$ and $\mathrm{f} 2$, there is no way to determine beforehand which fit factor might perform better.Fortunately, with the sophistication of today's experimental design programs it is relatively easy to test models developed using several different response variables.

\section{Afterword}

Future work should evaluate the performance of other fit factors such as mean dissolution time
(MDT),Sd, Rescigno's index,etc. as response variables. The authors would like to receive feedback from those in the industry who may have encountered similar experiences or who can share experimental design data for this type of analysis.

Please send correspondence to: Henry H.Flanner Assistant Director, Drug Delivery Sciences Shire Laboratories, Inc. 1550 East Gude Drive Rockville, MD 20850

Phone:301-838-2634 Fax:301-838-2504

Email:hflanner@shirelabs.com

\section{References}

1.) M.C. Gohel and M.K. Panchal, "Comparison of In Vitro Dissolution Profiles" Pharmaceutical Technology 24(3): 92-102 (2000)

2.) V.P. Shah et al.,"In Vitro Dissolution Profile Comparison - Statistics and Analysis of the Similarity Factor $\mathrm{f2}$," Pharmaceutical Research 15:889-896 (1998).

3.) J.E. Polli, et al.,"Methods to Compare Dissolution Profiles and a Rationale for Wide Dissolution Specifications for Metorprolol Tartrate Tablets," J. Pharm.Sci. 86 (6), 690-700 (1997).

4.) C.F.Vesey and S.C. Porter, "Modified-Release Coating of Pellets with and Ethyl Cellulose Based Coating Formulation: Coating Process Considerations." 1999 AAPS Poster Session 